\title{
1 Alteration of Gut Microbiome in Lung Cancer Patients
}

2

\section{Li Ming, ${ }^{\text {a }}$ Yu Fang, ${ }^{\text {b }}$ Chen Xiaohui, ${ }^{\text {b }}$ Zhou Huan, ${ }^{\text {b }}$ Wei Xiaoqing, ${ }^{c}$ Liu Yinhui, ${ }^{a}$}

Liu Yuanyu, ${ }^{\mathrm{b}}$ Tang Li, ${ }^{\text {a }}$ Yuan Jieli, ${ }^{\mathrm{a} *}$ Wen Shu, ${ }^{\mathrm{a} *}$ and Chen Jun ${ }^{\mathrm{b}} *$

${ }^{\mathrm{a} C}$ College of Basic Medical Science, Dalian Medical University, Dalian, China.

${ }^{\mathrm{b}}$ Department of Medical Oncology, the Second Affiliated Hospital of Dalian Medical University, Dalian,China;

${ }^{\mathrm{c}}$ The Core Laboratory of Medical Molecular Biology of Liaoning Province, Dalian Medical University, Dalian, China.

*Correspondence:

Prof. Wen Shu, Ph.D. Department of Microecology, College of Basic Medical Science, Dalian Medical University, No.9 Western Section of Lvshun South Street, Lvsghhunkou District, Dalian, Liaoning 116044, China. E-mail: $\underline{\text { wsh2008cn@sina.com }}$

Prof. Yuan Jieli, M.D. Department of Microecology, College of Basic Medical Science, Dalian Medical University, No.9 Western Section of Lvshun South Street, Lvshunkou District, Dalian, Liaoning 116044, China. E-mail: zgwst@126.com Prof. Chen Jun, Ph.D. Department of Medical Oncology, The Second Affiliated Hospital, Dalian Medical University, 467 Zhongshan Road, Shahekou District, Dalian, Liaoning 116023, China. E-mail: chenjundl@ vip.sina.com 
bioRxiv preprint doi: https://doi.org/10.1101/640359; this version posted May 16, 2019. The copyright holder for this preprint (which was not certified by peer review) is the author/funder. All rights reserved. No reuse allowed without permission.

23 
ABSTRACT Lung cancer is the leading cause of cancer death. Better understanding of factors and pathways involved in lung cancer is needed to improve diagnose and treatment strategies. Recent studies have provided insights into the possible correlation between intestinal dysbiosis and cancer development. Although the immunological relationship between gut and lung had been suggested by many researches, however, to date, no study had investigated the characterization of gut microbiome in treatment naïve lung cancer patients, whether it is distinct from that of health individuals and contribute to the onset and development of lung cancer remain unclear. In this study, we investigated whether gut microbiome of lung cancer patients (LC, $n=28)$ is altered compare with that of matched healthy individuals $(\mathrm{HC}, \mathrm{n}=19)$ by high throughout sequencing of the V3-V4 regions of $16 \mathrm{~S}$ rDNA in their fecal samples. We also identified microbiota signatures specific for different histological types of lung cancer, including SSC, ADC, and SCLC. The gut microbiome of lung cancer patients is characterized by decreased relative abundance of Prevotella, and increased bacteria groups such as Actinomyces, and Streptococcus, etc. We also detected a mild structural shift in gut microbiome between ADC and SCLC patients. Our results showed that the gut microbiome of lung cancer patients altered significantly compared with healthy individuals. However, the association between microbial dysbiosis and lung cancer is not clearly understood, future studies involving larger cohorts and metagenomics, or metabolomics, may elucidate the correlations between gut microbiota and lung cancer development.

IMPORTANCE This is the first report to show the alteration of gut microbiome in 
bioRxiv preprint doi: https://doi.org/10.1101/640359; this version posted May 16, 2019. The copyright holder for this preprint (which was not certified by peer review) is the author/funder. All rights reserved. No reuse allowed without permission.

46 lung cancer patients. Our results showed that the gut microbiome of lung cancer

47 patients altered significantly compared with healthy individuals.

48

49 KEYWORDS: lung cancer, gut microbiome, dysbiosis, Prevotella 


\section{INTRODUCTION}

Lung cancer is the leading cause of cancer death (1). There are estimated 1.82 million new cases of lung cancer globally, which constitutes nearly $13 \%$ of all newly diagnosed cancer cases annually (2). More than one-third of lung cancer worldwide occurring in China, where 733,000 new cases of lung cancer are diagnosed, and about 591,000 Chinese people died from it each year (3-4). Better understanding of factors and pathways involved in lung cancer is urgently needed to improve treatment strategies.

As a heterogeneous disease, many factors are involved in the onset and development of lung cancer. Although smoking is considered as an important factor, only $10-15 \%$ of smokers develop cancer (5), which highlights other influences, such as the involvement of microbial communities. Recent studies showed that changes in the lung microbiome may be relevant for progression and exacerbations in lung cancer $(6,7)$. By comparison of microbiome in bronchoalveolar lavage fluid of patients with lung cancer with benign mass like lesions, Lee et al. found that the genera Veillonella and Megasphaera are more abundant in lung cancer patients, which may serve as potential biomarkers for the disease detection/classification (8). A recent research, in which surgical lung tissue samples were used, concluded that the microbiota of the lung cancer is unique, with the genus Thermus more abundant in tissue from advanced stage (IIIB, IV) patients, while Legionella is higher in patients who develop metastases (9). These studies provide insights into the possible correlation between microbiota and lung cancer development. 
However, lung microbiota doesn't seem to be the only microbial factor contributing to the development of lung cancer. The influence of gut microbiota on lung immunity has been vastly explored and several studies have linked changes in the gut microbiome with lung diseases $(10,11)$. For example, gut flora is responsible for inducing lung inflammatory reaction against bacterial challenge and enhancing neutrophils infiltration through TLR4 in mice $(12,13)$. Antibiotic treatment can cause overgrowth of particular fungal species in the gut and promote allergic airway inflammation via fungi-induced prostaglandin E2 (14). Conversely, the lung microbiota also influences the gut microbiota through the blood stream. Acute lung injury (ALI) can disrupt the lung microbiota, induces a transient translocation of bacteria into blood and causes an acute increase of bacterial load in cecum (15). These studies emphasized the important role of gut-lung axis in development of diseases (16). However, to date, no study had investigated the characterization of gut microbiome in treatment naïve lung cancer patients, whether it is distinct from that of health individuals and contribute to the onset and development of lung cancer remain unclear.

In this study, we investigated whether gut microbiome of lung cancer patients (LC, $n=28)$ is altered compare with that of matched healthy individuals (HC, $n=19)$ by high throughout sequencing of the V3-V4 regions of $16 \mathrm{~S}$ rDNA in their fecal samples. We also identified microbiota signatures specific for different histological types of lung cancer, including SSC, ADC, and SCLC.

\section{RESULTS}


Characteristics of participants. A total of 50 participants enrolled in the Department of Medical Oncology, The Second Affiliated Hospital of Dalian Medical University (Dalian, China). Participants were excluded after fecal sample collection because of antibiotic use, received chemotherapy, or combined with other diseases such as diabetes mellitus (Fig. 1). Finally, a total of 28 LC patients were remained, they were mainly males (83\%) with a median age of 65 years old (Table 1) and with an average smoking index of $606.79 \pm 127.1 .19$ healthy controls (HC) were included for age and gender matching, with an average smoking index of $350.00 \pm 72.1$ (P > 0.05 compared with LC group) (details in Table 1). More than $64 \%$ of the enrolled LC patients were smokers, with a high smoking index about 606.79 \pm 127.1 . Among them, $28.57 \%(8 / 28)$ of the patients were diagnosed as SCLC, and $71.43 \%(20 / 28)$ were NSCLC. Among the NSCLC patients, $21.43 \%$ (6/28) were diagnosed as squamous cell carcinoma and 50.00\% (14/28) were adenocarcinoma.

The overall structure of gut microbiome in LC patients. By sequencing of the 16S ribosomal RNA gene, we found that The HC and LC groups share about 2368 same Operational Taxonomic Units (OTUs), but there are 372 OTUs were obtained specifically in the HC group, and 202 were obtained specifically in the LC group (Fig. 2A). We observed significant decrease in alpha diversity of gut microbiota in LC group, which expressed by the ACE and Chao1 index (Fig. 2B and C). Whereas the Shannon diversity index and the Simpson index did not show significant differences between two groups (Fig. S1). Changes in the relative abundance of gut microbes in LC patients were observed not only on the phylum level, but also on the levels of 
117 order, class, and family (Fig. S4). At genus level, significant decreased abundance of

118 Prevotella and elevated abundance of Bacteroides, and Ruminococcus etc. were

119 detected in LC patients (Fig. 2D).

The beta diversity metrics from the control and LC individuals also showed strong grouping pattern. Although significant inter-individual variation exists among patients and the healthy controls, the fecal microbiota of the two groups still separated clearly according to community composition using Principal component analysis (PCA, Fig. S3), and unweighted/weighted UniFrac Principal coordinates analysis (PCoA) (Fig. 2E). These differences were also observed by the two-dimensional

Nonmetric Multidimensional Scaling (NMDS) based on unweighted/weighted UniFrac (Fig. 2F). Most of the samples from each group clustered together as evaluated by Hierarchical clustering based on Weighted UniFrac by the method of Unweighted pair-group method with arithmetic means (UPGMA, Fig. S4). Especially, when analyzed by the method of Partial Least Squares Discriminant Analysis (PLSDA), we observed a significant separation between the LC patients and HCs (Fig. $2 \mathrm{G})$. microbiome in LC patients was further proved by the LEfSe approach, which identified the key phylotypes responsible for the difference between the two groups.

137 which were most abundant in the LC group, and Prevotella, Bacteroidetes, and 
contributed to the difference between the intestinal microbiota of LC patients and HCs (Fig. 3A and B). The significantly elevated relative abundance of Actinomyceae, Streptococcus, and Ruminococcus, and decreased abundance of Prevotellaceae were observed in the gut microbiome of most of the LC patients, which suggested a highly consistence among different individuals (Fig. S5).

Using the method of Metastats, we found a significant decrease in the abundance of Bacteroidetes (phylum), Bacteroidia (class), Bacteroidales (order) and elevated abundance of Firmicutes (phylum), Bacilli (class), Actinomycetales (order), Bacillales (order), Lactobacillales (order) in gut of the LC patients (Fig. S6). On the family level, significant elevation of the relative abundance of Streptococcaceae, Actinomycetaceae, decreased abundance of the Prevotellaceae and Veillomellaceae were observed in gut of LC patients compared with HCs (Fig. 4A). These changes may mainly due to the changing of genus such as the Streptococcus, Actimomyces, and Prevotella etc (Fig. 4B). In addition, we also detected elevation of genera such as Ruminococcus, Rothia, Bacillus, Peptostreptococcus, Mycoacterium, etc, and decreased abundance of Dialister in gut of LC patients, which are consistent with LEfSe analysis results.

\section{Comparison of gut microbiome in LC patients with specific histological types.}

We further performed a detailed comparison of the gut microbiome in lung cancer patients according to different histological types, including adenocarcinoma (Group A, $\mathrm{n}=14$ ), squamous cell carcinoma (Group B, n=6), and SCLC (Group C, n=8). Only the index of alpha diversity (ACE) in SCLC patients is significantly lower than control (Fig. 5A). Other groups showed no significant differences compared either with HC 
161

162

163

or other groups, although the average indexes of each of the specific histological type are obviously lower than that of the HCs (Fig. 5A and Fig. S7). The beta diversity analysis by PCoA and NMDS showed no obvious separation between groups (Fig. 5B and C). When analyzed by the method of PLSDA, we observed a mild separation between group A and C, which suggested that the gut microbiome of SCLC patients may differ from that of LC patients with adenocarcinoma (Fig. 5D). The taxonomy-based comparison at the genus level showed that, Bifidobacterium, Clostridium, and Prevotella, etc, are the dominant phylotypes in group A, but were significantly reduced in the other two groups. In group B, the dominant genera including Ruminococcus, Lachnospira, and Lactobacillus, etc, which are less abundant in group A and C. in addition, the genera of Streptococcus, Anaerotruncus, and Bacillus, etc are more abundant in group $\mathrm{C}$ when compared with group A and B (Fig. S8).

\section{DISCUSSION}

Alterations of the gut microbiome influence the incidence and progression of not only gastric carcinogenesis (17), but also extra-intestinal cancers, such as breast and hepatocellular carcinoma, presumably through inflammatory and metabolic circuitries (18). Meanwhile, gut microbiota was also found contribute to the acute lung injury (19), the exacerbation of chronic obstructive pulmonary disease (COPD) (20), and the development of asthma (21), which highlighted its important role in affecting the respiratory system. Actually the hypothesis of "gut - lung" axis has been raised 20 
183

years ago, when study found that gut-derived injurious factors can reach to the lung and systemic circulation via the intestinal lymphatics (22). Gut flora was found to be responsible for inducing lung inflammation against bacteria in mice and enhancing neutrophils infiltration through activation of tool like receptor 4 (TLR4) (23). Such immune transmission from gut to lung has been proved by many studies. One viewpoint supports that exacerbation of chronic lung diseases occur as an uncontrolled and inappropriate inflammatory response to bacteria colonizing damaged airways due to an ineffective Peyer's patch-derived T lymphocyte response (24). These studies above strongly suggested a possible correlation between intestinal dysbiosis and the development of lung cancer. However, to our knowledge, no study has explored this possibility yet.

Although based on a small number of cases, we found a statistically significant decrease of alpha diversity in gut microbiota of lung cancer patients, compared with that of the healthy controls, which was consist with previous discovery that non-malignant lung tissues have higher microbiota alpha diversity than the paired tumors (10). The decline in both the bacterial diversity and richness was found in gut of patients with chronic inflammation such as inflammatory bowel disease (IBD) and colonrectal cancer, especially in patients with conventional adenoma (25). Reduced respiratory microbiome was also shown associated with greater emphysema and increased immune cell infiltration in COPD patients (26). These observations seem in line with the hygiene hypothesis that diverse microbes play an essential role in establishing the immune networks of a host, while in patients with various 
non-communicable inflammatory diseases, such as asthma, these regulatory networks seemed to be underrepresented and poorly developed (27).

Alterations of the gut microbial structure in lung cancer patients were characterized by the significant decrease of Bacteroidetes (phylum), Bacteroidia (Class), Bacteroidales (Order), and increase of Firmicutes (phylum), Bacilli (Class), Actinomycetales, and Bacillales (Order), which were mainly contributed by the reduction of bacteria genera such as Prevotella and Dialister, and the increase in Ruminococcus, Streptococcus, Rothia, Bacillus, Actinomyces, Peptostreptococcus, etc. These changes are not consistent with previous studies in sliva, bronchoalveolar lavage fluid, or lung tissues in patients with lung cancer, except that the genus of Streptococcus was previously found increased in lower airway of LC patients (28). But interestingly, they are highly agreed with the observations in patients with colon cancer that the class of Bacilli, genera of Streptococcus, Actinomyces, Peptostreptococcus, and etc are enriched (29). Importantly, we found a significantly increased ratio of Bacteroides/Prevotella, which was also proved in patients with colorectal cancer when compared with normal individuals (25). Taken together, these results indicate a state of dysbiosis in the gut microbiome of patients with lung cancer. As the major genus that was found significantly reduced in LC patients' gut, Prevotella had drawn many of our attentions. Prevotella strains are classically considered commensal bacteria due to the extensive presence in the healthy human body and the rare involvement in infections. Prevotella can stimulate epithelial cells to produce cytokines such as IL-8, IL-6 and CCL20, which can promote mucosal 
Th17 immune responses and neutrophil recruitment (30). Increased Prevotella abundance is associated with augmented Th17-mediated mucosal inflammation, which is in line with the marked capacity of Prevotella in driving Th17 immune responses through activation of TLR2 (30). These studies suggested an immune-stimulating activity of this genus. Congruously, Prevotella abundance was found reduced within the lung microbiota of patients with asthma and COPD (31), which further highlighted its important role in affecting lung cancer development.

On the other side, the increasing of certain bacteria in LC patients suggested a deleterious role of them in the development of cancer. Species of Streptococcus are found to increase in patients with LC. Compared to healthy controls, the NSCLC patients presented significantly higher frequencies of Th1 and Th17 cells reacting to $S$. salivarius and $S$. agalactiae, in the PB, LC, and GI tract (32). The order of Actinomycetales had been as potential colorectal cancer driver bacteria (25). Among them, the filamentous Gram-positive anaerobic genus Actinomyces, was found to cause Actinomycosis, which is a rare and slowly progressive infectious disease that can affect a variety of organ systems including the lung (33). Actinomyces was also found significantly associated with the carcinoma-in-adenoma group (34). Besides, Peptostreptococcus species, P. stomatis was confirming known to be associated with CRC (35). P. anaerobius, which is increased in human colon tumors compared with nontumor tissues, can enhance AOM-induced tumorigenesis in mice by activating TLR2/4-ROS-cholesterol axis (36). In addition, species of Atopobium, A. parvalum was found positively correlates with pediatric IBD disease severity (37). 
However, the effects of these bacteria on lung cancer development are still unclear, which deserves in-deep study.

In addition, we compared the gut microbiome of lung cancer patients according to different histological types based on collected samples, including SSC, ADC, and SCLC. Although the alpha diversity among groups showed no difference, we detected an obvious separation of beta diversity between patients with ADC and SCLC. Given the limited number of study cases, further large scale studies on the characterization of gut microbiome in LC patients with different histological types are necessary.

Conclusions. In conclusion, this is the first report to show the alteration of gut microbiome in lung cancer patients. Our results showed that the gut microbiome of lung cancer patients altered significantly compared with healthy individuals. However, the association between microbial dysbiosis and lung cancer is not clearly understood, future studies involving larger cohorts and metagenomics, or metabolomics, may elucidate the correlations between gut microbiota and lung cancer development.

\section{MATERIALS AND METHODS}

Study subjects and sample collection. The recruitment of participants and the process of sample collection are depicted in Fig. 1. Fifty patients (age, $50-75$ years) were ultimately recruited from the Second Affiliated Hospital of Dalian Medical University, Dalian, China, from September 2015 to July 2016. Fecal samples were collected in Stool Collection Tubes, which were pre-filled with Stool DNA Stabilizer for collection (Stratec, Germany), then frozen and stored at $-80{ }^{\circ} \mathrm{C}$ for further use. All 
subjects were examined clinically before sampling and were subsequently divided into three groups: SCLC $(n=17), \operatorname{AC}(n=18), \operatorname{SCC}(n=15)$. The samples of the healthy controls $(\mathrm{HC}, \mathrm{n}=30)$ were collected during routine physical examination at the First Affiliated Hospital of Dalian Medical University, Dalian, China.

The participants with the following diseases were excluded: cardiovascular disease, diabetes mellitus, liver cirrhosis, irritable bowel syndrome, inflammatory bowel disease, infections with known active bacteria, fungi, or virus. Those who abused drug or alcohol in the last year, or used antibiotics, probiotics, prebiotics, or synbiotics in the month, or received chemotherapy before collection of the fecal sample were also excluded.

DNA extraction, polymerase chain reaction (PCR) and pyrosequencing. The microbial genome was extracted using E.Z.N.A. ® Stool DNA kit (Omega Bio-tek, Inc.) according to the manufacturer's instructions. A Nanodrop 2000 Spectrophotometer was used to evaluate the purity and concentration of isolated DNA. The polymerase chain reaction (PCR) to amplify the V3-V4 region of bacterial $16 \mathrm{~S}$ ribosomal RNA gene was performed as described previously. After amplicons extraction, samples were purified using AXYGEN gel extraction kit (Qiagen) and quantified by Quant-iT PicoGreen dsDNA Assay Kit on Microplate reader (BioTek, FLx800). Sequencing and data analysis were subsequently performed on an Illumina MiSeq platform by Personal Biotechnology Co., Ltd. (Shanghai, China). The taxa classification and statistical analysis were conducted as described in previous studies. Statistics and analysis. Illumina MiSeq sequences obtained after quality control 
analysis were used in the present analysis, which were uploaded to QIIME (Quantitative Insights Into Microbial Ecology, v1.8.0) for further study. The operational taxonomy units (OTUs) of representative sequences at a similarity cutoff of $97 \%$ and their relative abundance ( $\alpha$-diversity) were used to calculate ACE and Chao1 index by UCLUST. The abundance and diversity of the OTUs ( $\beta$-diversity) were examined using principal component analysis (PCA), Principal coordinates analysis (PCoA) and nonmetric multidimensional scaling (NMDS) with weighted and unweighted UniFrac analysis in $\mathrm{R}$ software. The statistical significance of the separation among groups was assessed by the linear discriminant analysis effect size (LEfSe) method based on linear discriminant analysis scores exploited by Curtis Huttenhower (http://huttenhower.sph.harvard.edu/galaxy/), which used the nonparametric factorial Kruskal-Wallis and Wilcoxon rank sum test to identify key OTUs for separating different treatment groups at a significance level of 0.05 . Metastats analysis was performed based on the raw count data matrix to find out the taxa statistically different between HC and LC samples.

For the analyses of clinical data, the non-parametric $t$-test between HC and LC groups was performed with the assistance of GraphPad Prism 6 (Graph Pad Software, La Jolla, CA, USA). Results were considered to be statistically significant with $\mathrm{P}<$ 0.05. The taxa classification and statistical analysis were conducted as described in previous studies (38).

Ethics statement. This study protocol was approved by the Ethics Committee of the Second Affiliated Hospital of Dalian Medical University, Dalian, China. After 
bioRxiv preprint doi: https://doi.org/10.1101/640359; this version posted May 16, 2019. The copyright holder for this preprint (which was not certified by peer review) is the author/funder. All rights reserved. No reuse allowed without permission.

315 receiving a written description of the aim of this study, all participants gave written

316 informed consent prior to enrollment.

317

318 


\section{SUPPLEMENTARY MATERIAL}

320

321

322

323

324

325

326

327

328

\section{Supplementary Figure legends}

FIG S1, Comparison of the Shannon (A) and Simpson (B) index of gut microbiome in HC and LC groups.

FIG S2, The gut bacterial composition in different groups at Phylum, Class, Order, and Family levels.

FIG S3, Principal component analysis of gut microbiome in HC and LC groups. PC1 and PC2 account for $73.08 \%$ of the variation.

FIG S4, Hierarchical clustering based on Weighted UniFrac by the method of UPGMA. Number of samples: HC, 2-20, LC, 62-98

FIG S5, The relative abundance of the most differentially abundant bacterial taxons identified by LEfSe in each samples of different among groups.

FIG S6, The specific bacterial Phyla, Classes, and Orders that are significantly changed in LC patients compared with HCs detected by the MetaStat method.

FIG S7, Alpha diversity of gut microbiota in LC patients with specific histological types. (A) Venn diagram of shared and independent bacterial OTUs in different groups. (B) ACE, (C) Chao1, (D) Shannon, (E) Simpson.

FIG S8, The taxonomy-based comparison among groups at the genus level showed by heat map.

\section{ACKNOWLEDGMENTS}

This study was supported by the National Natural Science Foundation of China 
341 (NSFC, 81370113), the Nature Science Foundation of Liaoning Province, China

342 (2015020262), and the Research Foundation from the Department of Education,

343 Liaoning Province, China (L2016003). This work was supported by Liaoning

344 Provincial Program for Top Discipline of Basic Medical Sciences.

346 CONFLICTS OF INTERSTS

347 The authors declare no competing interest. 


\section{REFERENCES}

1. Herbst RS, Heymach JV, Lippman SM. 2008. Lung cancer. N Engl J Med 359(13): 1367-1380.

2. Siegel RL, Miller KD, Jemal A. Cancer statistics, 2016. 2016. CA Cancer J Clin 66(1): 7-30.

3. Youlden DR, Cramb SM, Baade PD. 2008. The international epidemiology of lung cancer: geographical distribution and secular trends. J Thorac Oncol 3(8): 819-831.

4. Wang P, Zou J, Wu J, Zhang C, Ma C, Yu J, Yu J, Zhou Y, Li B, Wang K. 2017. Clinical profiles and trend analysis of newly diagnosed lung cancer in a tertiary care hospital of East China during 2011-2015. J Thorac Dis 9(7): 1973-1979.

5. Wang X, Pittman GS, Bandele OJ, Bischof JJ, Liu G, Brothers JF 2nd, Spira A, Bell DA. 2017. Linking polymorphic p53 response elements with gene expression in airway epithelial cells of smokers and cancer risk. Hum Genet 133(12): 1467-1476.

6. Man WH, de Steenhuijsen Piters WA, Bogaert D. 2017. The microbiota of the respiratory tract: gatekeeper to respiratory health. Nat Rev Microbiol 15(5): 259-270.

7. Roudi R, Mohammadi SR, Roudbary M, Mohsenzadegan M. 2017. Lung cancer and beta-glucans: review of potential therapeutic applications. Invest New Drugs 35(4): 509-517. 
8. Lee SH, Sung JY, Yong D, Chun J, Kim SY, Song JH, Chung KS, Kim EY, Jung JY, Kang YA, Kim YS, Kim SK, Chang J, Park MS. 2016. Characterization of microbiome in bronchoalveolar lavage fluid of patients with lung cancer comparing with benign mass like lesions. Lung Cancer 102: 89-95.

9. Yu G, Gail MH, Consonni D, Carugno M, Humphrys M, Pesatori AC, Caporaso NE, Goedert JJ, Ravel J, Landi MT. 2016. Characterizing human lung tissue microbiota and its relationship to epidemiological and clinical features. Genome Biol 17(1): 163.

10. Chen MM, Zahs A, Brown MM, Ramirez L, Turner JR, Choudhry MA, Kovacs EJ. 2014. An alteration of the gut-liver axis drives pulmonary inflammation after intoxication and burn injury in mice. Am J Physiol Gastrointest Liver Physiol 307(7): G711-718.

11. Trompette A, Gollwitzer ES, Yadava K, Sichelstiel AK, Sprenger N, Ngom-Bru C, Blanchard C, Junt T, Nicod LP, Harris NL, Marsland BJ. 2014. Gut microbiota metabolism of dietary fiber influences allergic airway disease and hematopoiesis. Nat Med 20(2): 159-166.

12. Zou Y, Dong C, Yuan M, Gao G, Wang S, Liu X, Han H, Li B. 2014. Instilled air promotes lipopolysaccharide-induced acute lung injury. Exp Ther Med 7(4): 816-820.

13. Ben DF, Yu XY, Ji GY, Zheng DY, Lv KY, Ma B, Xia ZF. 2012. TLR4 mediates lung injury and inflammation in intestinal ischemia-reperfusion. $\mathrm{J}$ 
14. Kim YG, Udayanga KG, Totsuka N, Weinberg JB, Núñez G, Shibuya A. 2014. Gut dysbiosis promotes M2 macrophage polarization and allergic airway inflammation via fungi-induced PGE(2). Cell Host Microbe 15(1): 95-102.

15. Sze MA, Tsuruta M, Yang SW, Oh Y, Man SF, Hogg JC, Sin DD. 2014. Changes in the bacterial microbiota in gut, blood, and lungs following acute LPS instillation into mice lungs. PLoS One 9(10): e111228.

16. He Y, Wen Q, Yao F, Xu D, Huang Y, Wang J. 2017. Gut-lung axis: The microbial contributions and clinical implications. Crit Rev Microbiol 43(1): $81-95$.

17. Huang X, Li C, Li F, Zhao J, Wan X, Wang K. 2018. Cervicovaginal microbiota composition correlates with the acquisition of high-risk human

18. Coker OO, Dai Z, Nie Y, Zhao G, Cao L, Nakatsu G, Wu WK, Wong SH,

19. Mima K, Nakagawa S, Sawayama H, Ishimoto T, Imai K, Iwatsuki M, Hashimoto D, Baba Y, Yamashita YI, Yoshida N, Chikamoto A, Baba H. 2017.

20. Nicod LP, Kolls JK. 2015. Chair's summary: mechanisms of exacerbation of lung diseases. Ann Am Thorac Soc 12 Suppl 2: S112-114. 

Gut, microbiota-dependent trimethylamine-N-oxide is associated with long-term all-cause mortality in patients with exacerbated chronic obstructive pulmonary disease. Nutrition 45:135-141.e1.

422

22. Kang YB, Cai Y, Zhang H. 2017. Gut microbiota and allergy/asthma: From pathogenesis to new therapeutic strategies. Allergol Immunopathol (Madr) 45(3): 305-309.

23. Magnotti LJ, Upperman JS, Xu DZ, Lu Q, Deitch EA. 1998. Gut-derived mesenteric lymph but not portal blood increases endothelial cell permeability and promotes lung injury after hemorrhagic shock. Ann Surg 228(4): 518-527.

24. Tsay TB, Yang MC, Chen PH, Hsu CM, Chen LW. 2011. Gut flora enhance bacterial clearance in lung through toll-like receptors 4. J Biomed Sci 18: 68.

25. Samuelson DR, Welsh DA, Shellito JE. 2015. Regulation of lung immunity and host defense by the intestinal microbiota. Front Microbiol 6: 1085.

26. Peters BA, Dominianni C, Shapiro JA, Church TR, Wu J, Miller G, Yuen E, Freiman H, Lustbader I, Salik J, Friedlander C, Hayes RB, Ahn J. 2016. The gut microbiota in conventional and serrated precursors of colorectal cancer. Microbiome 4(1): 69.

27. Richmond BW, Brucker RM, Han W, Du RH, Zhang Y, Cheng DS, Gleaves L, Abdolrasulnia R, Polosukhina D, Clark PE, Bordenstein SR, Blackwell TS, Polosukhin VV. 2016. Airway bacteria drive a progressive COPD-like phenotype in mice with polymeric immunoglobulin receptor deficiency. Nat 
28. Smits HH, Hiemstra PS, Prazeres da Costa C, Ege M, Edwards M, Garn H, Howarth PH, Jartti T, de Jong EC, Maizels RM, Marsland BJ, McSorley HJ,

29. Liu HX, Tao LL, Zhang J, Zhu YG, Zheng Y, Liu D, Zhou M, Ke H, Shi MM, Müller A, Pfefferle PI, Savelkoul H, Schwarze J, Unger WW, von Mutius E, Yazdanbakhsh M, Taube C. 2016. Microbes and asthma: Opportunities for intervention. J Allergy Clin Immunol137(3): 690-697. cancer (CRC) patients. PLoS One 6(1): e16393.

32. Hilty M, Burke C, Pedro H, Cardenas P, Bush A, Bossley C, Davies J, Ervine

31. Larsen JM. 2017. The immune response to Prevotella bacteria in chronic inflammatory disease. Immunology 151(4): 363-374. microbial communities in asthmatic airways. PLoS One 5(1): e8578.

33. Ma QY, Huang DY, Zhang HJ, Wang S, Chen XF. 2017. Upregulation of bacterial-specific Th1 and Th17 responses that are enriched in CXCR5 (+) CD4 (+) T cells in non-small cell lung cancer. Int Immunopharmacol 52: 305-309. 
34. Laguna S, Lopez I, Zabaleta J, Aguinagalde B. 2017. Actinofmycosis associated with foreign body simulating lung cancer. Arch Bronconeumol 53(5): 284-285.

35. Kasai C, Sugimoto K, Moritani I, Tanaka J, Oya Y, Inoue H, Tameda M, Shiraki K, Ito M, Takei Y, Takase K. 2016. Comparison of human gut microbiota in control subjects and patients with colorectal carcinoma in adenoma: Terminal restriction fragment length polymorphism and next-generation sequencing analyses. Oncol Rep 35(1): 325-333.

36. Yu J, Feng Q, Wong SH, Zhang D, Liang QY, Qin Y, Tang L, Zhao H, Stenvang J, Li Y, Wang X, Xu X, Chen N, Wu WK, Al-Aama J, Nielsen HJ, Kiilerich P, Jensen BA, Yau TO, Lan Z, Jia H, Li J, Xiao L, Lam TY, Ng SC, Cheng AS, Wong VW, Chan FK, Xu X, Yang H, Madsen L, Datz C, Tilg H, Wang J, Brünner N, Kristiansen K, Arumugam M, Sung JJ, Wang J. 2017. Metagenomic analysis of faecal microbiome as a tool towards targeted non-invasive biomarkers for colorectal cancer. Gut 66(1): 70-78.

37. Tsoi H, Chu ESH, Zhang X, Sheng J, Nakatsu G, Ng SC, Chan AWH, Chan FKL, Sung JJY, Yu J. 2017. Peptostreptococcus anaerobius induces intracellular cholesterol biosynthesis in colon cells to iduce proliferation and causes dysplasia in mice. Gastroenterology 152(6): 1419-33 e5.

38. Mottawea W, Chiang CK, Mühlbauer M, Starr AE, Butcher J, Abujamel T, Deeke SA, Brandel A, Zhou H, Shokralla S, Hajibabaei M, Singleton R, Benchimol EI, Jobin C, Mack DR, Figeys D, Stintzi A. 2016. Altered 
bioRxiv preprint doi: https://doi org/101101/640359; this version posted May 16, 2019. The copyright holder for this preprint (which was not certified by peer review) is the author/funder. All rights reserved. No reuse allowed without permission. 
bioRxiv preprint doi: https://doi.org/10.1101/640359; this version posted May 16, 2019. The copyright holder for this preprint (which was not certified by peer review) is the author/funder. All rights reserved. No reuse allowed without permission.

TABLE 1 Characteristics of the study population

\begin{tabular}{|c|c|c|c|}
\hline Variables & Healthy controls & Patients with lung cancer & $p$-value \\
\hline Sample size & $\mathrm{n}=19$ & $\mathrm{n}=28$ & \\
\hline Age & $57.21 \pm 1.706$ & $61.50 \pm 1.345$ & 0.0525 \\
\hline Gender (F/M) & $3 / 19$ & $6 / 28$ & 0.6385 \\
\hline $\operatorname{BMI}\left(\mathrm{kg} / \mathrm{m}^{2}\right)$ & $24.57 \pm 1.427$ & $25.95 \pm 1.025$ & 0.0831 \\
\hline Smoking index & $350.00 \pm 72.1$ & $606.79 \pm 127.1$ & 0.0845 \\
\hline \multicolumn{4}{|l|}{ Final diagnosis } \\
\hline & & SCLC $8(28.57 \%)$ & \\
\hline & & Extensive/limited stage $5 / 3$ & \\
\hline & & NSCLC $20(71.43 \%)$ & \\
\hline & & Squamous cell carcinoma $6(21.43 \%)$ & \\
\hline & & Stage IIA/IIB/IIIA/IIIB/IV 1/1/1/1/2 & \\
\hline & & Adenocarcinoma 14 (50.00\%) & \\
\hline & & Stage IIA/IIIA/IIIB/IV 2/2/1/9 & \\
\hline
\end{tabular}

489 


\section{FIGURE LEGEND}

FIG 1 The recruitment of participants and the process of sample collection.

FIG 2 The intestinal bacterial composition in HCs and LC patients. (A) Venn diagram of shared and independent bacterial OTUs in HC and LC groups. (B) Comparison of the ACE and Chao1 index of HC and LC groups. (C) The bacterial composition in different groups at Genus level. (D) Principal Coordinate Analysis (PCoA) based on weighted Unifrac distances among different samples. (E) NMDS based on weighted Unifrac distances among different samples. (F) PLS-DA of the gut microbiome in HC and LC patients.

FIG 3 LEfSe analysis of gut microbiota in HC and LC groups. (A) LEfSe identified the most differentially abundant bacterial taxons among groups. Group-specific enriched taxa are indicated with a positive LDA score bar with different colors. Only taxa meeting an LDA significant threshold $>2$ are shown. (B) Taxonomic cladogram obtained from LEfSe analysis of $16 \mathrm{~S}$ rDNA sequences. The brightness of each dot is proportional to its effect size.

FIG 4 The specific bacterial groups that are significantly changed in LC patients compared with HCs detected by the MetaStat method. (A) Families, (B) Genera.

FIG 5 Comparison of gut microbiome in LC patients with specific histological types.
(A) Comparison of the ACE index of different groups. (B) PCoA based on unweighted and weighted Unifrac distances among different samples. (C) NMDS based on unweighted and weighted Unifrac distances among different samples. (D) PLS-DA of the gut microbiome in LC patients with specific histological types. 
A

$\square \mathrm{HC} \square \mathrm{LC}$

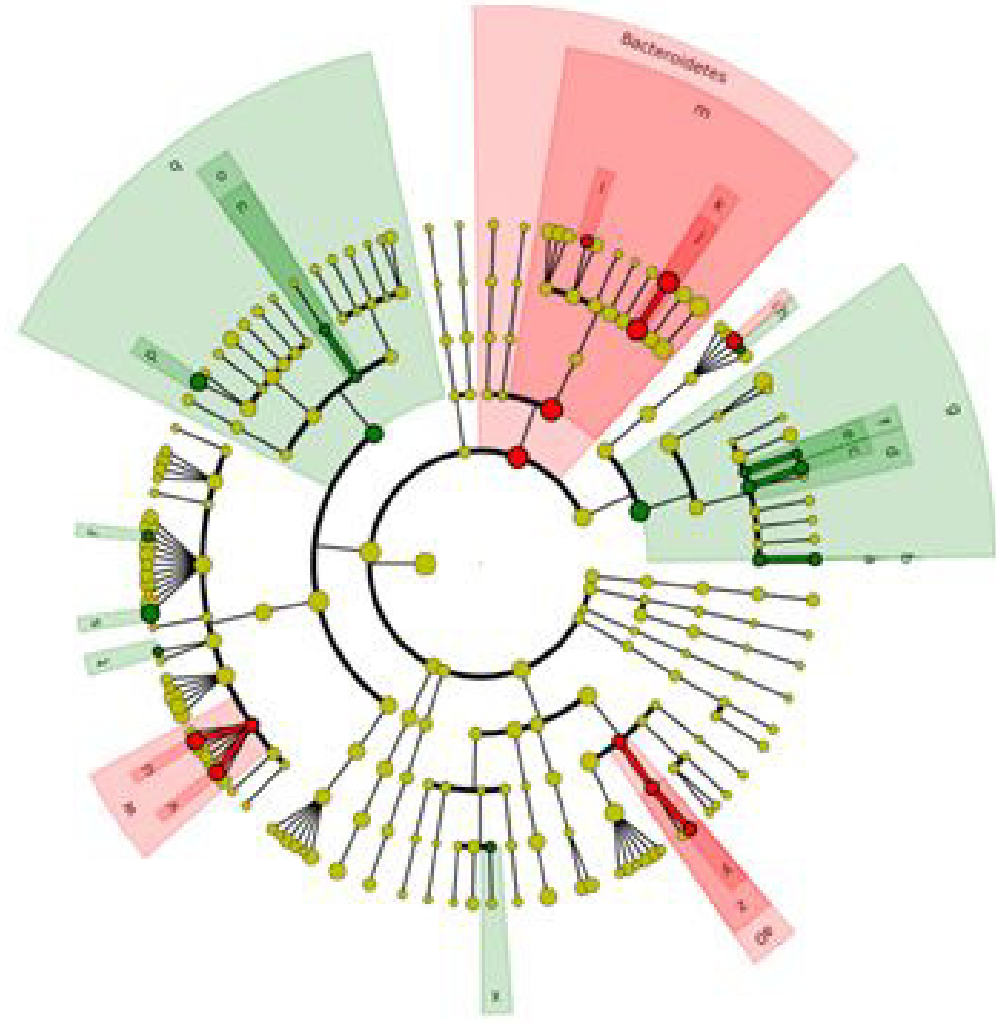

$\square$ a: Actinomyces

$\square$ b: Actinomycetaceae

c: Rothia

d: Micrococcaceae

$\square$ e: Mycobacterium

$\square$ f: Mycobacteriaceae

$\square$ g: Actinobacteria

h: Atopobium

i: Collinsella

j: Prevotella

k: Prevotellaceae

1: Odoribacter

m: Bacteroidia

n: Gemellaceae

o: Gemellales

$\square$ p: Streptococcus

q: Bacill

r: Clostridium

$\square$ s: Ruminococcus

t: Peptostreptococcus

u: Dialister

v: Phascolarctobacterium

w: Veillonellaceae

x: Methylobacteriaceae

y: Haemophilus

z: Pasteurellaceac

ao: Pasteurellales

B

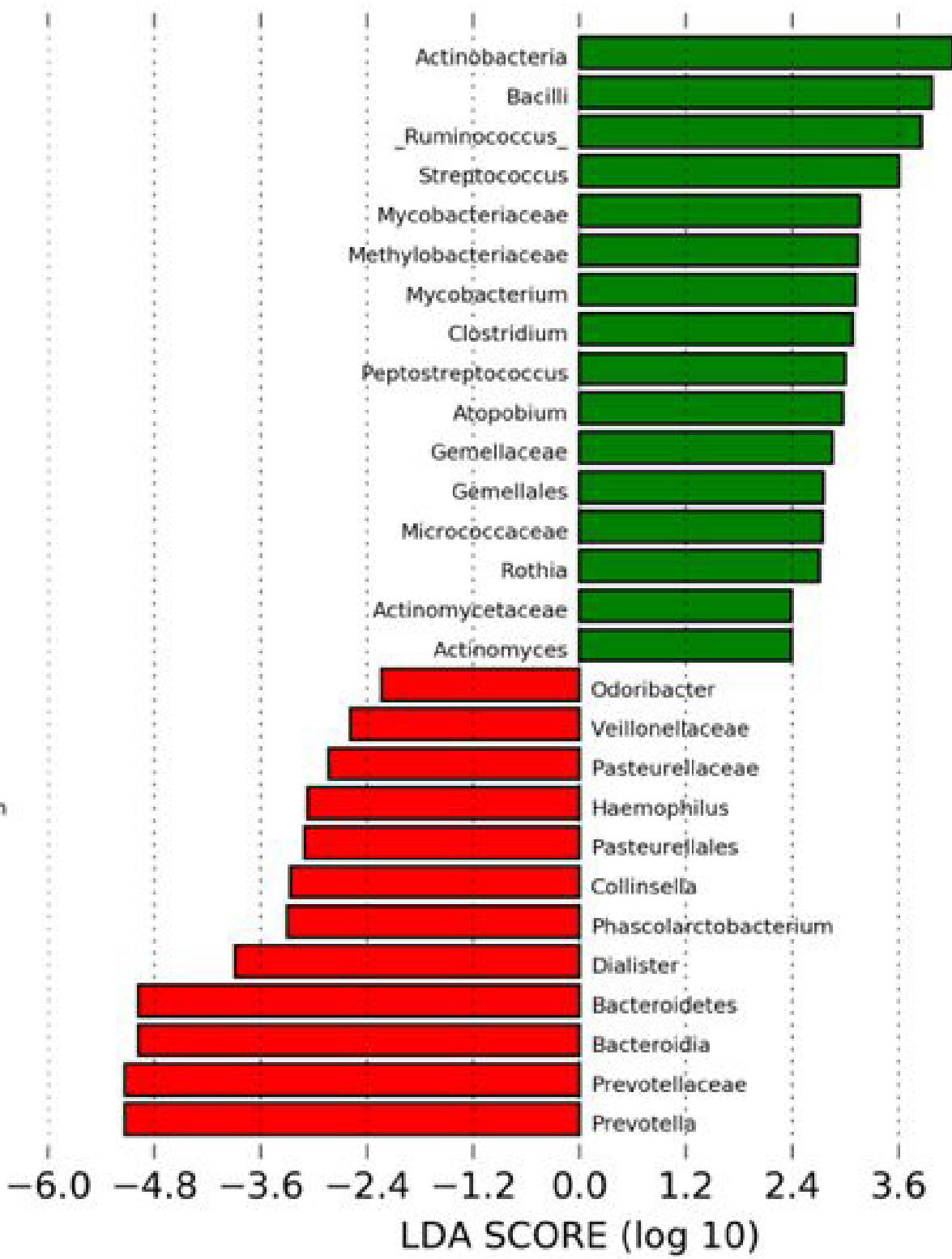

4.8 
A
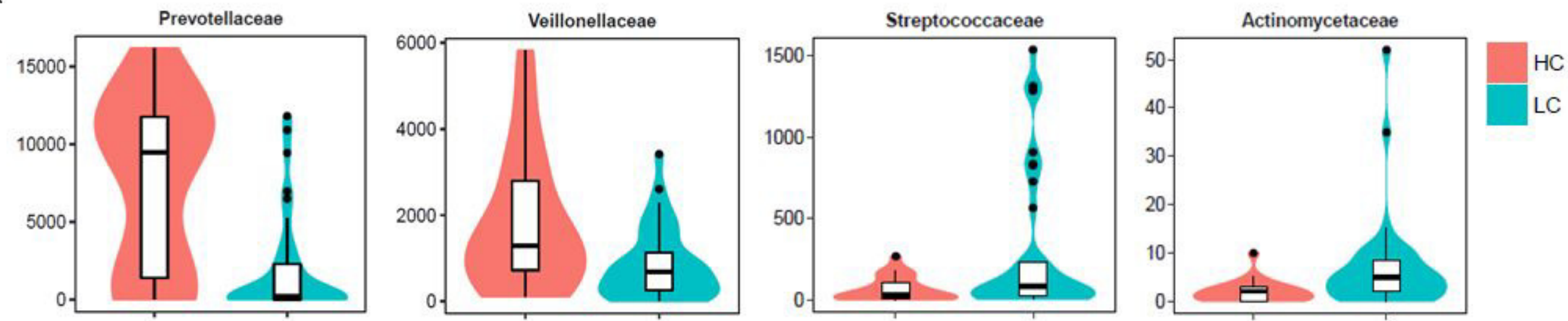

B
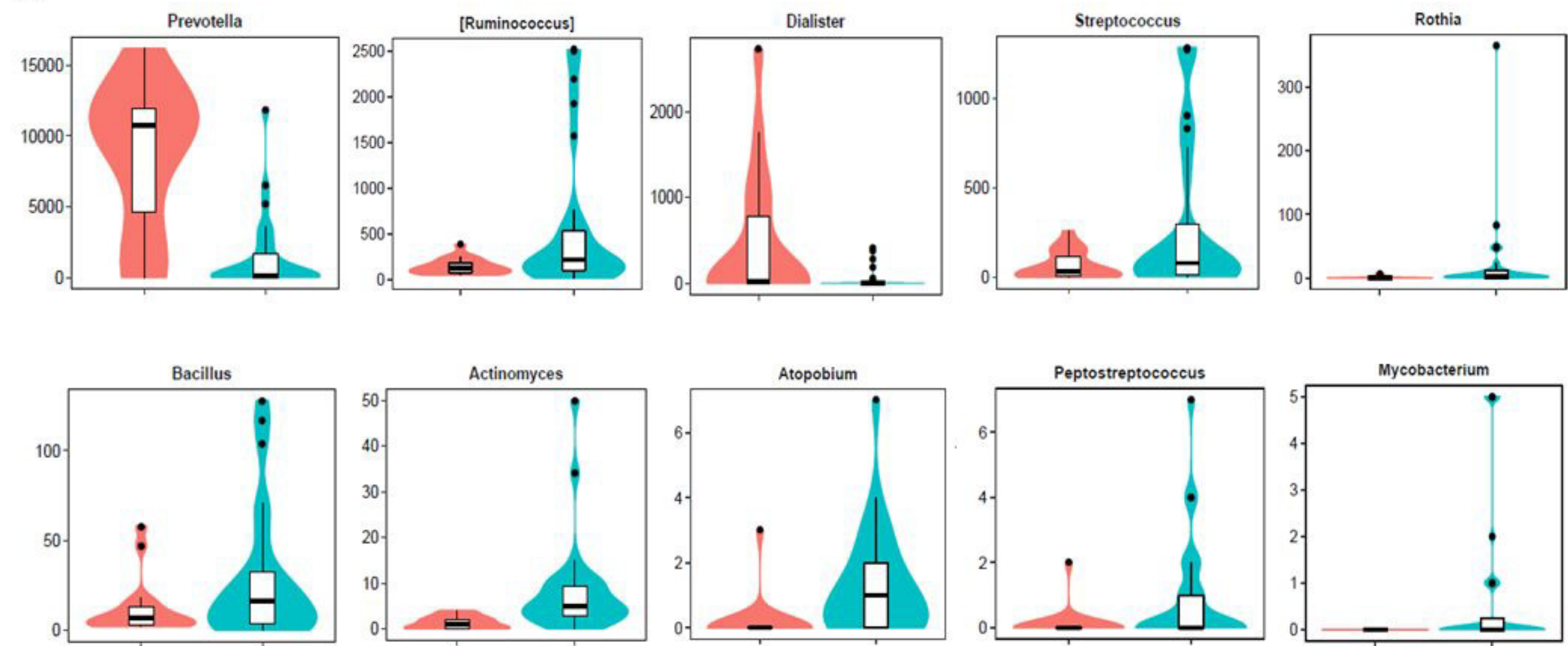
A

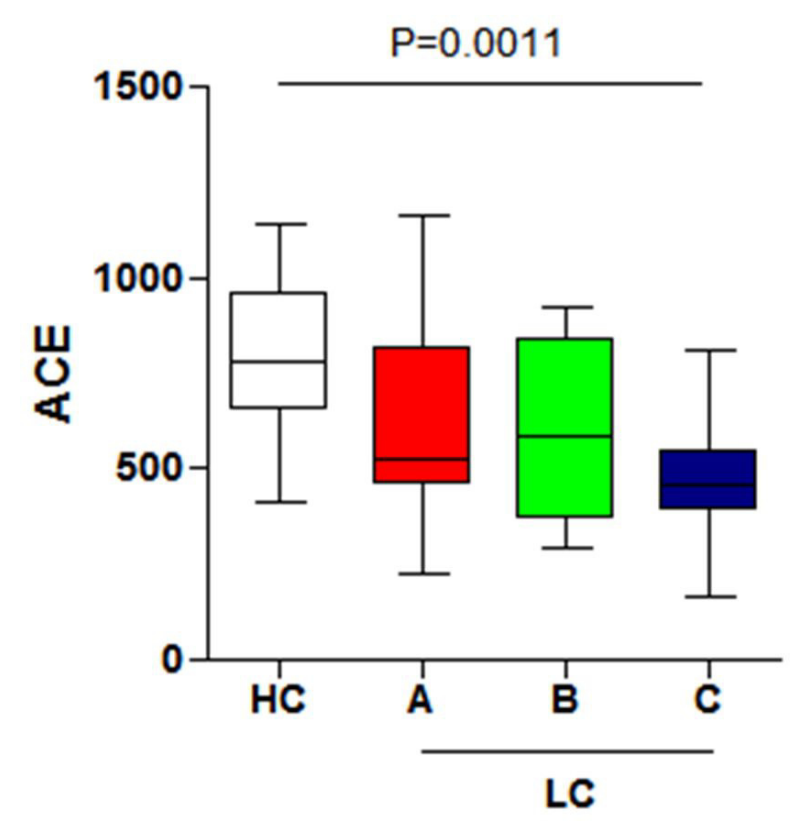

C

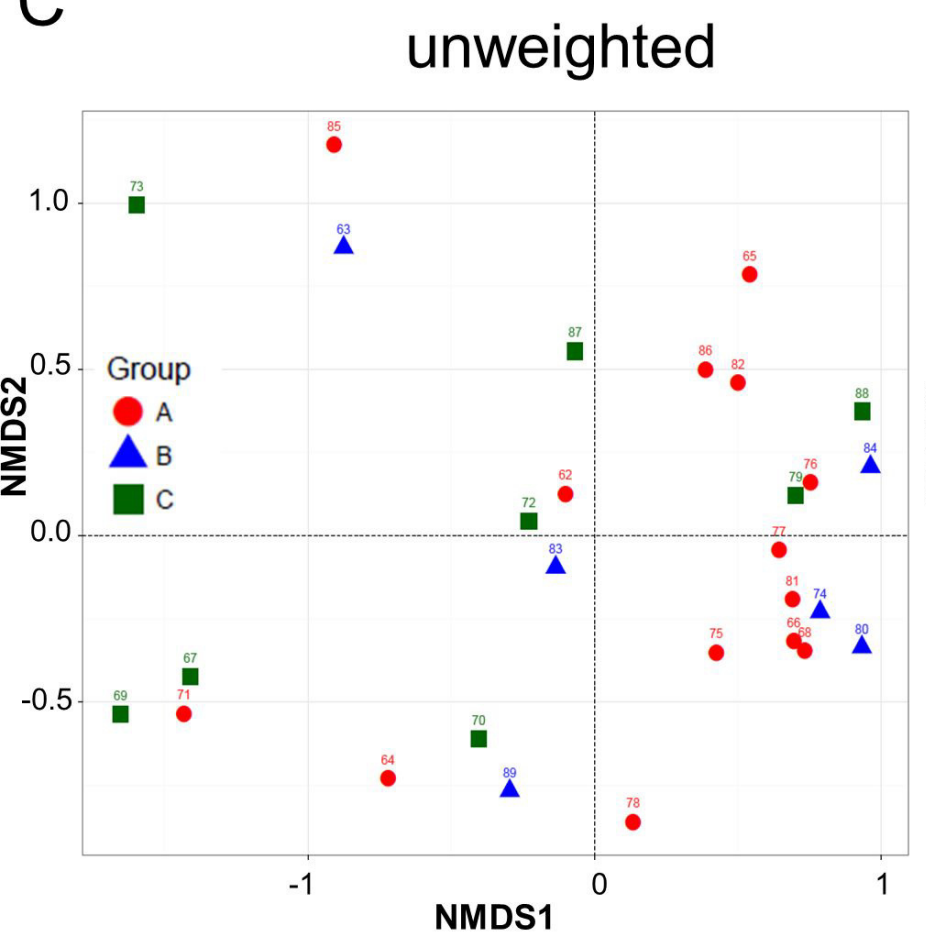

B

unweighted
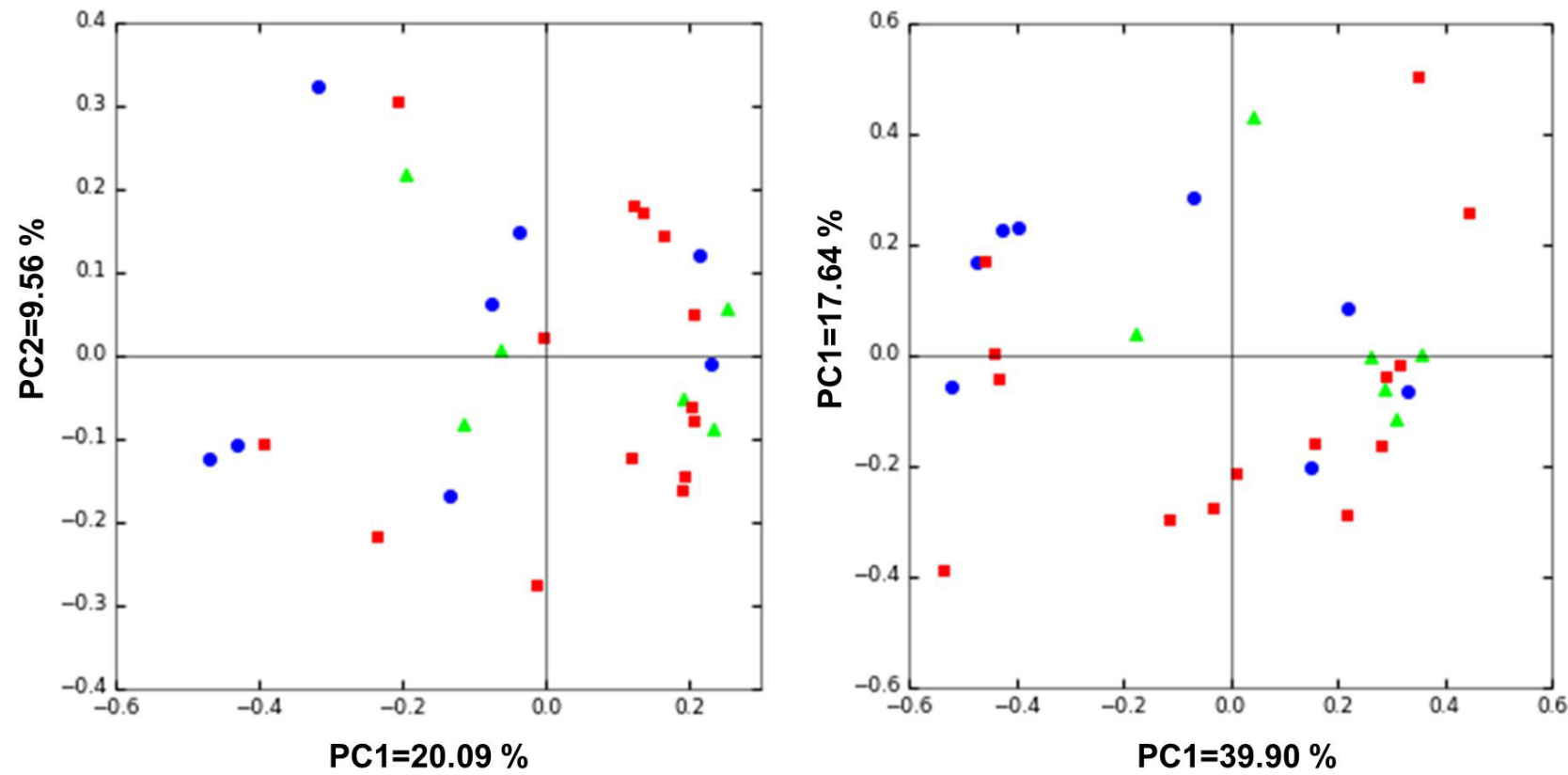

D

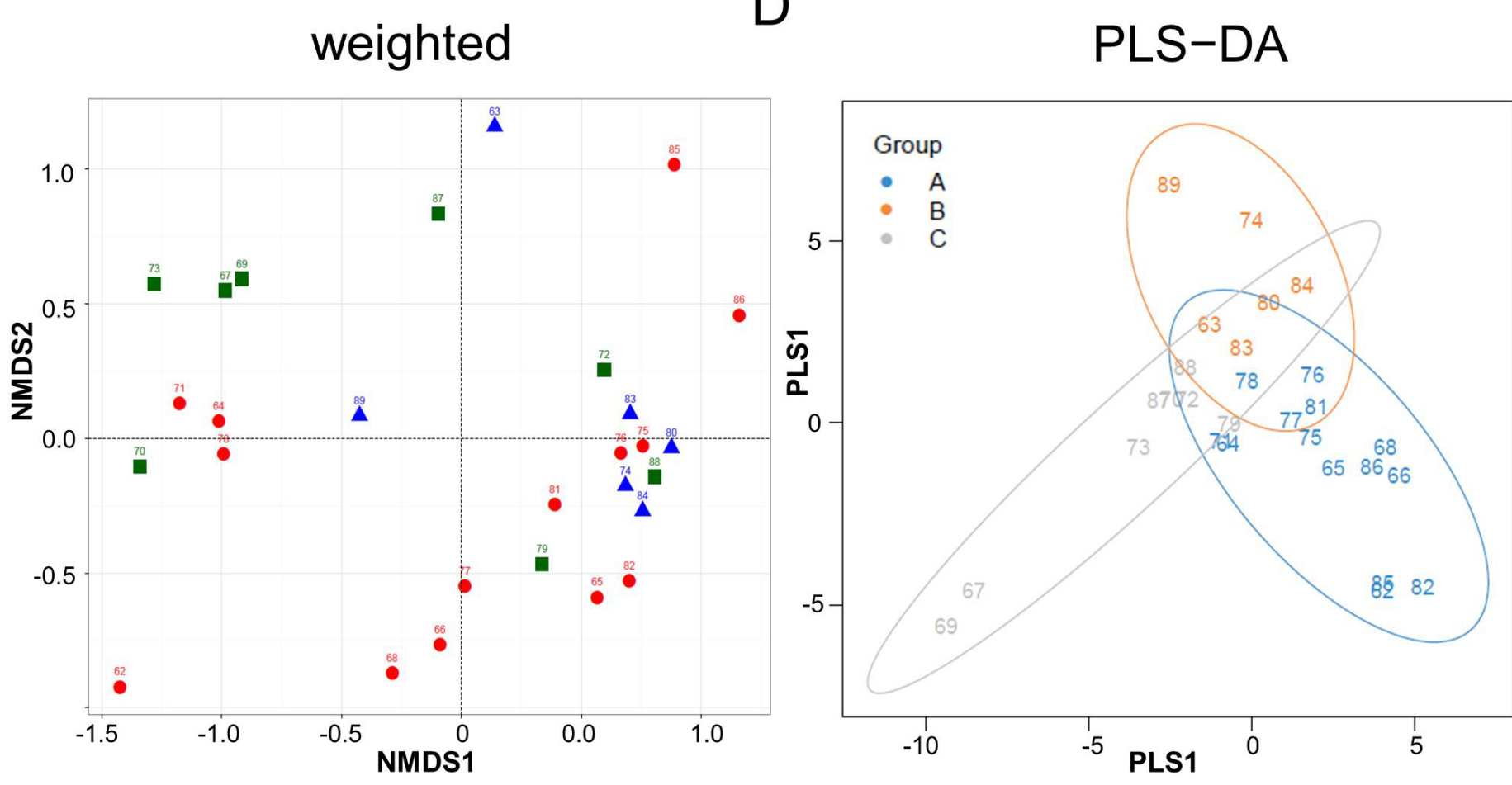

A

$\triangle B$

- $\mathrm{C}$ 